\title{
EPIDEMIOLOGIA DE LA LEPRA A TRAVÉS DEL ESTUDIO DE LA FRECUENTACIÓN EN EL HOSPITAL ESPECIALIZADO DE TRILIO DURANTE EL PERÍODO 1943-1995
}

\author{
Juan Román Urbina Torija (1), M. ${ }^{a}$ del Pilar García Salazar (2) M. de las Mercedes Letón Pastor \\ (2) y Raquel Ruiz Pérez (2). \\ (1) Delegación de Sanidad. Junta de Comunidades Castilla la Mancha. \\ (2) Escuela Universitaria de Enfermería de Guadalajara.
}

\section{RESUMEN}

Fundamento: El estudio pretende describir el perfil sociodemográfico y clínico del enfermo de lepra hospitalizado y comprobar si su tipología ha cambiado en la historia del centro.

Métodos: Se trata de un estudio descriptivo y retrospectivo mediante la revisión de una muestra de las historias clínicas abiertas en el centro desde su origen en 1943 hasta 1995 . Sc eligieron mediante muestreo aleatorio sistemático 366 historias. de las que se cumplimentó un cuestionario que recogía variables sociodemográficas y clínicas.

Resultados: La mayoría de los enfermos lueron varones (71.9\%. IC: 67.3-76.4). de edad media de 39.6 años (IC: 37.941.4) solteros (46.2\%, IC: 41-51.3) con bajo nivel cultural (analfabetismo: $54.1 \%$. IC: $40-69$ ) y una ocupación relacionada con la agricultura $(35.5 \%$. IC:30.6-40.4) en regiones del sur de España (enfermos procedentes de Andalucía 52.8\%. IC: 45.854). La enfermedad presentó antecedentes familiares en un 31.1\% (IC:26.4-35.9) de los afectados y formas clínicas graves multibacilares (lepra lepromatosa $66.1 \%$, IC:61.2-71) que afectaba a miembros inferiores en un $72.1 \%$. cabeza en el $63.1 \%$ y miembros superiores $64.4 \%$. La mortalidad global fue de $31.1 \%$ en los ingresados (IC: $26.4-35.9$ ). A lo largo del período de estudio el enfermo ingresado ha envejecido y suavizado su clínica y mortalidad. La estancia hospitalaria ha sido de 7.1 años (IC: 6.1-8.1) en general, aunque al final del estudio se aproxima a tiempos de ingreso menores. acordes con la duración del tratamiento de la enfermedad (2.2 años).

Conclusiones: Un varón jóven, con economía precaria y residente en el sur de nuestro país, parece ser el perfil del enfermo. que con formas anatomoclínicas severis inicialmente y una evolución posterior favorable, puede representar esta enlermedad en un país con una endenia autóctona y una epidemiología característica. que está en fase de pre-erradicación.

Palabras clave: Lepra. Epidemiología. Pertil clínico.
Correspondencia:

Juan Ramón Urbina Torija

Delegación de Sanidad

C/ Fernández Iparaguirre. 1

19001 Guadalajara

Fax: 949216865.

\section{ABSTRACT \\ Leprosy Epidemiology through the Study of Frequency of Visits in Trillo Specialised Hospital over the period 1943-1995}

Background: The purpose of this study is to describe the social-demographic and clinical profile of hospitalised leprosy patients and to check whether typology has changed during the history of the centre.

Methods: Descriptive and retrospective study, performed by means of reviewing a sample of the patient records registered at the Centre since it was founded in 1943 until 1995. 366 cases were chosen by means of a systematic random sample and questionnaires completed on social-demographic and clinical variables.

Results: Most of the patients were male $(71.9 \%$, IC: $67.3-$ 76.4), young (39.6 years of age, IC: $37.9-41.4$ ), single ( $46.2 \%$, IC: $41-51.3 \%$ ) with low levels of education (illiteracy: $54.1 \%$, IC: $40-69)$ with occupations relating to farming $(35.5 \%$, IC: 30.6-40.4) from Southern Spanish regions (patients from Andalucía $52.8 \%$, IC: $45.8-54$ ). The disease showed a family background in $31.1 \%$ of cases (IC: $26.4-35.9$ ) and serious inultibacillary forms (Lepromatose Leprosy $66.1 \%$, IC: $61.2-71$ ), which affected lower limbs in $72.1 \%$ of cases, heads in $63.1 \%$ and upper limbs in $64.4 \%$. Global mortality of people admitted to hospital was $31.1 \%$ (IC: 26.4-35.9). During the surveyed period, patient age increased, and symptoms and mortality decreased. In general, hospital stays were for long periods (7.1 years, IC: $6.1-8.1$ ), although at the end of the surveyed period, stays decreased considerably, in accordance with the duration of treatment (2.2 years).

Conclusions: A Young male, in a precarious financial situation living in the South of Spain appear to be the patient profile which, with severe initial anatomical symptoms and later favourable results may represent the disease in a country with an autochthonous endemy and characteristic epidemiology, which is in the pre-eradication phase.

Key words: Leprosy. Epidemiology. Clinical profile. 


\section{INTRODUCCIÓN}

La lepra fue la primera enfermedad infecciosa cuyo germen se aisló en el hombre (1873), y de las más antiguas citadas en la Historia de la Medicina - India 1400 años A.C. ${ }^{1}$ - Fue la más temida en la época preinfecciosa - se enterraba en vida a los leprosos con una frase lapidaria: «sic MORTUUS mundo.vivus iterum deo», «Estás muerto en este mundo solo vivirás con Dios $^{2}-$, y todavía hoy sigue siendo una enfermedad que afecta gravemente al cuerpo del paciente y a la mente de la comuni$\mathrm{dad}^{3}$. Aunque es prevalente en los trópicos, sin ser la más grave ni la más frecuente, es la que más estigmatización social origina ${ }^{4}$.

Es una enfermedad transmisible producida por el Mycobacterium Leprae que puede afectar a todos los tejidos del organismo, excepto al sistema nervioso central, con especial tropismo por las células de Schwann y las del sistema retículo endotelial. La afectación de piel, tejido conjuntivo y sistema nervioso periférico, hace que curse frecuentemente con lesiones llamativas y deformidades graves e irrecuperables en los miembros ${ }^{5,6}$. Esto y su cronicidad hacen de la enfermedad un problema de salud pública muy relevante para los 1.600 millones de habitantes que viven en los países donde la prevalencia es superior a 1 caso por 1.000 habitantes (datos correspondientes a $1987)^{6,7}$.

Su incidencia en países y áreas subdesarrolladas hace difícil el registro de casos y su tratamiento. En 1966 se conocían 2.850 .000 casos, en 1976 se registraron 3.600 .000 , en 1986 fueron $5.350 .000^{7} \mathrm{y}$ en 1996 se han notificado $926.259^{8}$. Actualmente, después de la introducción de la quimioterapia múltiple por la $\mathrm{OMS}^{7,9}$, y desde 1990, se creen curados cerca de 8 millones de personas, con una cobertura de un $91 \%$ de población leprosa controlada ${ }^{8}$. A pesar de que su distribución es muy desigual - Guinea, Sudán y Madagascar no consiguen bajar su prevalencia, el $68 \%$ de los casos se dan en la India y el 12\% en Brasil-, en el mundo ha descendido su incidencia anual a 500.000 casos, y su prevalencia está por debajo del millón de enfermos registrados $^{8}$. En España con 406 casos registrados y 5 casos nuevos al año ${ }^{10}$, estamos en una situación de pre-erradicación con una tasa de prevalencia de 0.1 por 10.000 habitantes, por debajo de 1 caso por 10.000 habitantes que constituye el Objetivo de Salud Para Todos en el año 2000 ${ }^{10}$.

\section{Introducción histórica}

La enfermedad fue importada a Occidente desde la India y, desde entonces y hasta el siglo XIX, se extendió por todo el mundo Occidental llegando a documentarse a finales de la Edad Media 18.000 leproserías en toda Europa ${ }^{1}$ en las distintas áreas endémicas. En España tres pueblos fueron los principales importadores, creándose cuatro focos endémicos que han persistido a lo largo de la historias: Fenicios (Galicia), Romanos (Levante y Centro) y Arabes (Andalucía y Canarias). El aislamiento y cuidado del leproso en «Leproserías» para ricos y «Casas del Leproso» para pobres, estuvo a cargo de la Iglesia hasta el Renacimiento (siglo XVI), cuando los Reyes Católicos crearon la figura de «Alcalde de la Lepra», civil experto en cuidados asistenciales ${ }^{12}$.

De cualquier manera, el período científico en la historia de la enfermedad no comienza hasta el siglo XIX, cuando el noruego Hansen aisló el germen causal durante un programa contra la enfermedad en su, entonces, endémico país. En 1897 durante el I Congreso de Berlín se establece la naturaleza infecciosa de la enfermedad y se inicia el registro exhaustivo de los casos, la búsqueda de antibióticos (En Estados Unidos durante 1943 Faget descubre la dapsona) ${ }^{7}$, aparecen hospitales medicoquirúrgicos y de investigación - 1903 Fontilles y 1943 Trillo-2 ${ }^{2}$, se establece una clasificación anatomoclínica de la enfermedad -VI Conferencia de Madrid de 1953 - y se inician Planes Nacionales de 
Intervención ${ }^{5}$. Se consigue el aislamiento del germen en animales de experimentación (Shepard en 1960) ${ }^{6}$, y aparecen pautas de poliquimioterapia para evitar la resistencia a la Dapsona como primer antibiótico' ${ }^{13}$.

Aunque al aparecer tratamiento antibiótico el control de la enfermedad se podría hacer ambulatoriamente ${ }^{14}$, la dificultad del seguimiento de los tratamientos, las recidivas y secuelas, y el todavía rechazo social a la enfermedad, mantuvieron los Centros Especializados.

El Instituto Leprologico de Trillo se afincó sobre un balneario del siglo XVIII, famoso en la zona por la capacidad curativa de las aguas de sus fuentes para enfermedades $\tan$ frecuentes entonces como la escrófula, reuma, lepra, etc. En 1770 el propio rey Carlos III inauguró un balneario que tuvo tantos éxitos curativos en un siglo, que en 1872 tuvo que ser remodelado y ampliado ante la asistencia de miles de bañistas. Sin embargo, la decadencia de estos centros durante el siglo XX y la Guerra Civil hicieron que el centro permaneciera abandonado durante los años cuarenta ${ }^{15}$.

Probablemente, el aumento de casos en la postguerra española, y la nccesidad de tener un centro sanitario cercano al centro geográfico y con una mínima inversión económica, hicieron posible la puesta en marcha de este sanatorio el 15 de diciembre de 1943․ El momento de su apertura y su situación estratégica creemos que puede aportar información respecto a la evolución y extensión de la enfermedad en los últimos 50 años en la comunidad.

Los objetivos en este estudio han sido:

1. Describir el perfil sociodemográfico y clínico del enfermo de lepra institucionalizado.

2. Describir la evolución en el tiempo de la tipología del enfermo.

\section{MATERIAL Y MÉTODOS}

\section{Diseño}

Se trata de un estudio descriptivo retrospectivo, realizado mediante la revisión de una muestra representativa del total de historias clínicas archivadas en el Instituto Leprologico Nacional de Trillo.

\section{Ámbito}

El pcríodo de estudio fue el comprendido entre los años 1943 y 1995. Se estudian los ingresos hospitalarios producidos durante dicho período. Esta institución es de ámbito nacional y tiene un carácter específico para esta enfermedad.

\section{Población de estudio}

Se eligieron 366 historias clínicas mediante muestreo sistemático aleatorio ${ }^{16}$ (arranque e intervalo aleatorio) del total de historias registradas (1.468 historias) en el intervalo 19431995. El tamaño muestral se definió para muestreos aleatorios simples de poblaciones finitas, mediante la aplicación informática del Programa EPIINFO-V6 STATCALC ${ }^{17}$. La muestra seleccionada mostró una proporcionalidad a la población diana de los sujetos ingresados para cada año de los estudiados ( $\mathrm{p}$-valor $<0,05$ ) (figura 1)

\section{Variables estudiadas}

\section{A) Sociodemográficas}

Sexo; Estado civil; Analfabetismo; Ocupación (se ha utilizado la Clasificación $\mathrm{Na}$ cional de Ocupaciones de $1994^{18}$, incluyendo las Amas de Casa en los parados); Emigración previa al ingreso en el centro, con periodos de estancia en otros países; Lugar de nacimiento: Provincia y Comunidad Autónoma; Lugar de procedencia: Se han registrado tanto la Comunidad Autónoma como la provincia donde residían los pacientes previamente a su ingreso; Edad al ingreso. 
Figura 1

Proporciôn de enfermos ingresados y elegidos cada año del estudio

\section{CASOS}

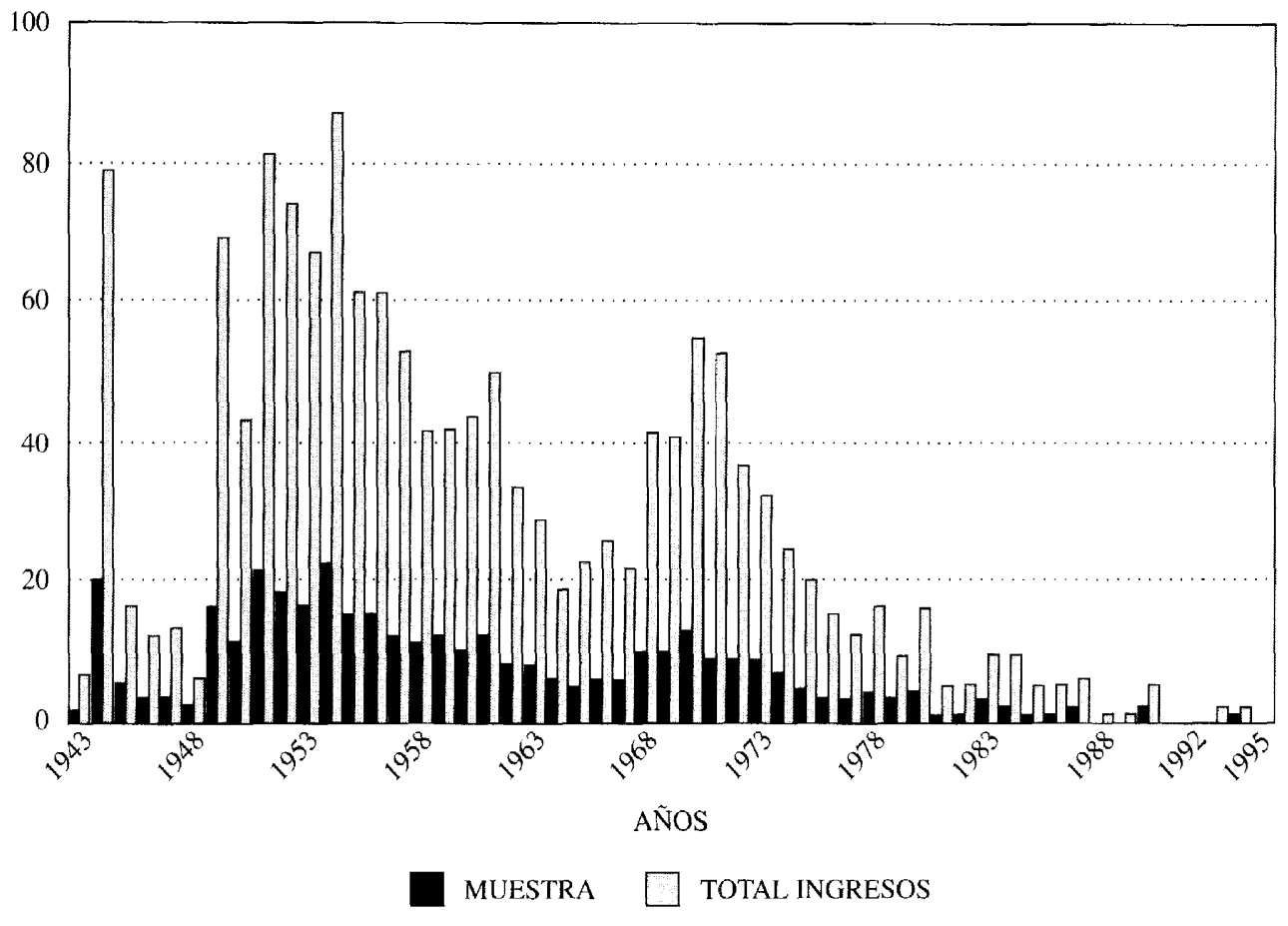

Nota: Porcentajes

B) Clínicas

Tipos de lepra: según Clasificación de Madrid-195319; clasificación topográfica: según partes del cuerpo; antecedentes familiares; causa de alta en el centro: se han considerado muerte por la enfermedad o sus complicaciones, transferencia o abandono del centro por cualquier causa distinta a la muerte, y no alta; estancia hospitalaria en años.

C) Períodos históricos de estudio

Acotamos tres períodos para facilitar el estudio de la frecuentación del centro:

Período 1.: Años 1943-1957. Máxima frecuentación del centro e introducción de la dapsona en España.
Período 2.: Años 1958-1972. Tendencia decreciente con oscilaciones e introducción de la clofazimina.

Período 3.: Años 1973-1995. Descenso de los ingresos hasta desaparecer, poliquimioterapia.

\section{Analisis estadístico y epidemiológico}

Las variables cualitativas se han estudiado con el parámetro chi cuadrado de Pearson. Las variables cuantitativas se han analizado con el análisis de la varianza de una vía y la prucba de t-Student.

El nivel de significación establecido para testar diferencias entre las distribuciones normales y de aproximación a la normal ha 
sido: error alfa tipo 1, p valor $<0.05$. Precisión de los Intervalos de Confianza para una seguridad de un $95 \%$.

\section{Registro e informatización}

Los datos fueron registrados en soporte informático con una base de datos DBASE III(+). El análisis estadístico se ha hecho en EPINNFO (6.2) y SPSS PC+ (4.0). Para el proceso de tex to y la representación gráfica de los resultados se han utilizado los programas Wordpcrfect (6.0) y Harvard Graphics (3.0).

Las bases de datos bibliográficas consultadas fueron Medline e Índice Medico Español (1990-1996).

\section{RESULTADOS}

Se han revisado un total de 366 historias clínicas, que suponen un $24,9 \%$ del total de las abiertas a lo largo de los 53 años de la existencia del centro.

\section{Aspectos sociodemográficos (tabla 1).}

El $71.9 \%$ de los enfermos han sido varones $(\mathrm{IC}=67.2-76.4)$, representando las mujeres el $28.1 \%(\mathrm{IC}=23.5-76.4)$. La media de edad de los estudiados ha sido de 39.68 años $(\mathrm{SD}=16.8$ ) con un Intervalo de Confianza de 37.9-41.4, siendo este valor de 40.4 en los varones $(\mathrm{SD}=16.4)$ y en las mujeres de 37.84 años ( $\mathrm{SD}=17.78)$. Las edades de 20-39 años comprenden el $48 \%$ de la población estudiada (figura 2).

En cuanto al estado civil se aprecia un predominio de la población soltera que representa el $46.2 \%$ (IC=41-52).

El analfabetismo es predominante en la población estudiada, $54.1 \% \quad(\mathrm{IC}=40-69)$,

Tabla 1

Variables sociodemográficas de los enfermos en el momento del ingreso (\%)

\begin{tabular}{|c|c|c|c|}
\hline & Varones & Mujeres & Total \\
\hline Edad (años) & 40,4 & 37,8 & 39,7 I.C. $: 38-41,4$ \\
\hline Antecedentes familiares & 35,1 & 44 & 31,1 I.C.:26.4-35,9 \\
\hline \multicolumn{4}{|l|}{ Estado civil } \\
\hline Solteros & 73,4 & 26,6 & 46,2 I.C.:41-51,3 \\
\hline Casados & 73,7 & 26,3 & 36,3 I.C.:31,4-41,3 \\
\hline Viudos & 50 & 50 & 8,2 I.C. $: 5,4-11$ \\
\hline Analfabetismo & 60,9 & 39,1 & 54,1 I.C.: $40-69$ \\
\hline \multicolumn{4}{|l|}{ Ocupación } \\
\hline Agricultura & 98,5 & 1,5 & 35,5 I.C. $: 30.6-40,4$ \\
\hline Parados & 17,8 & 82,2 & 19,9 I.C.:15-24 \\
\hline Construcción/minería & 100 & 0 & 10.1 I.C.: $7-13,2$ \\
\hline Emigración previa & 87.9 & 12,1 & 9 I.C.:6-11,9 \\
\hline \multicolumn{4}{|l|}{ Comunidad procedencia } \\
\hline Andalucía & 72,1 & 27,9 & 52,8 I.C. $: 43,8-54$ \\
\hline Madrid & 60 & 40 & 10.9 I.C. $7.7-14,1$ \\
\hline Valencia & 77,8 & 22,2 & 7.3 I.C. $: 4,3-10$ \\
\hline Galicia & 58,8 & 41,2 & 4,6 I.C.:2,5-6,8 \\
\hline Extremadura & 58,8 & 41,2 & 4,6 I.C.: $: 2,5-6,8$ \\
\hline \multirow[t]{2}{*}{ TOTALES } & 71,9 & 28.1 & \\
\hline & I.C.: $67,2-76,4$ & I.C.:23,5-32,7 & \\
\hline
\end{tabular}

1.C.: Intervalo de confianza $65 \%$ seguridad. 
Figura 2

Edad y sexo de los enfermos estudiados según tramos quinquenales

GRUPOS DE EDAD

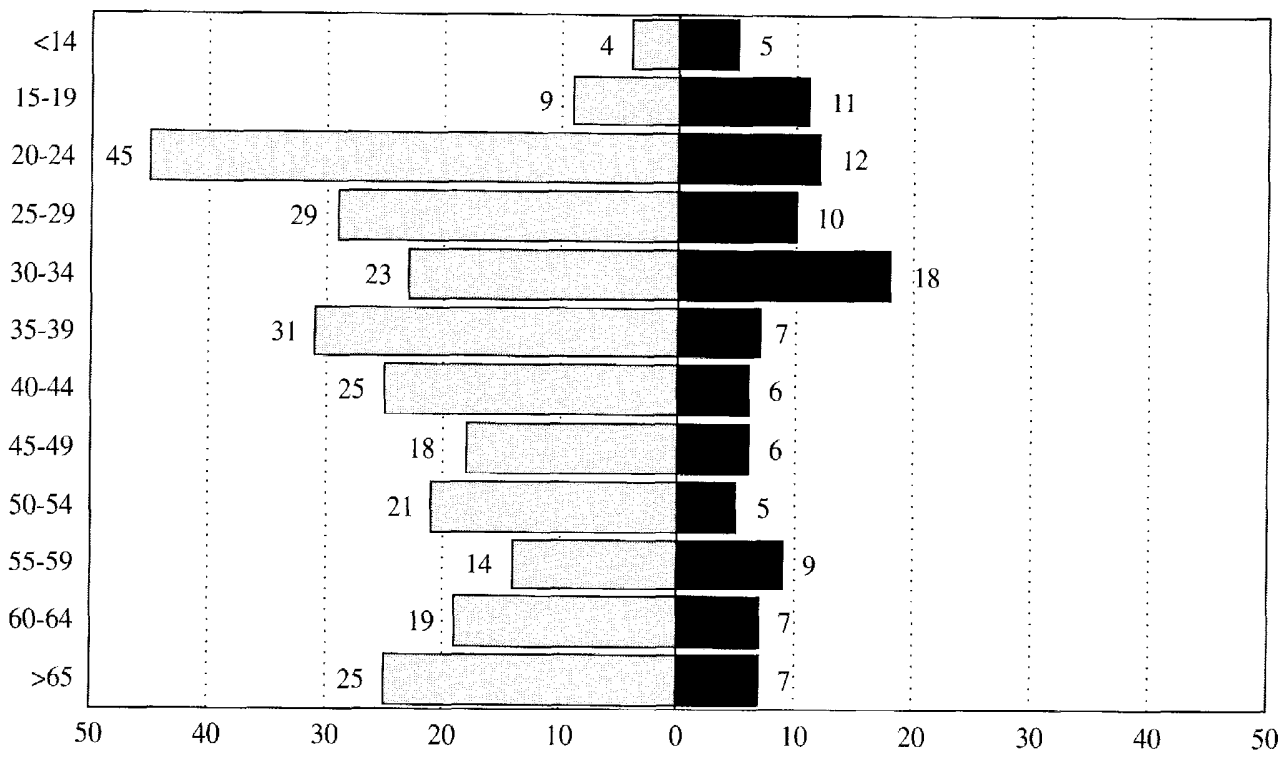

NUMERO DE CASOS

VARONES

MUJERES

Nota: Número absoluto de encuestados.

siendo desigual su distribución por sexos; varones $60.9 \%$ y mujeres $39.1 \%(\mathrm{p}<0.05)$. La agricultura es el sector de ocupación para la mayor parte de la población (35.5\% IC $=30.6-40.4)$, siendo los parados $(20 \%$ IC $=15.8-24)$ el segundo grupo mas frecuente (las amas de casa representan el $82.2 \%$ del total de parados) (figura 3 ).

El 13\% de la población estudiada había emigrado a otros países previamente a su ingreso, $87.9 \%$ varones.

Andalucía es la Comunidad Autónoma de nacimiento con mayor porcentaje de enlermos $(66 \%)$, siendo también la Comuni- dad con mayor número de residentes antes de su ingreso $(48.9 \%, \mathrm{IC}=43.8-54)$.

Otras comunidades donde los enfermos residían son: Madrid (11\%, IC=7.7-14), seguida de la Comunidad Valenciana $(7.4 \%$, IC $=4.7-10$ ) (figura 4).

La provincia que registra un mayor número de casos del total del país es Jaén con un $12.4 \%$ seguida de Madrid con el $11.8 \%$ y Málaga con un $11.5 \%$.

Las Comunidades de Andalucía, Madrid y Valencia aportan más varones que mujeres al centro. Sin embargo, Galicia y Extremadura no aportan diferencias significativas entre sexos. 
Figura 3

Ocupación de los enfermos elegidos en el momento del ingreso

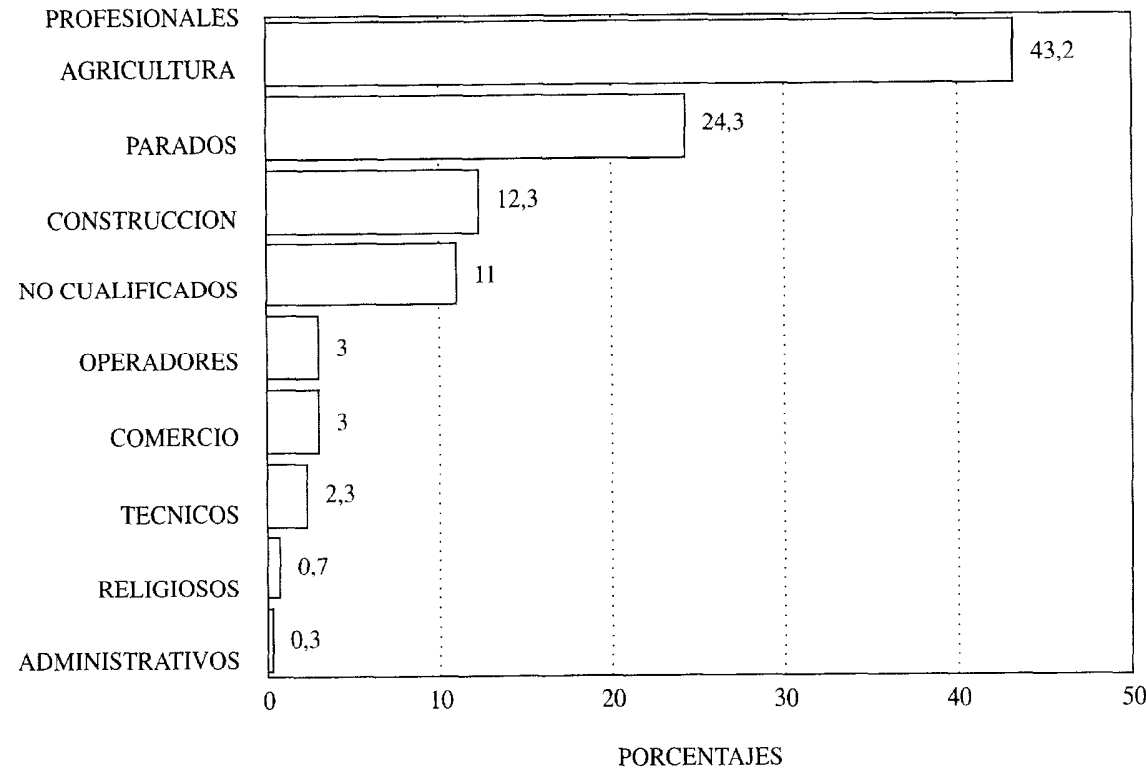

Nota: Porcentajes.

Figura 4

Ámbitos geográficos de procedencia de los enfermos al ingreso por Comunidad Autónoma

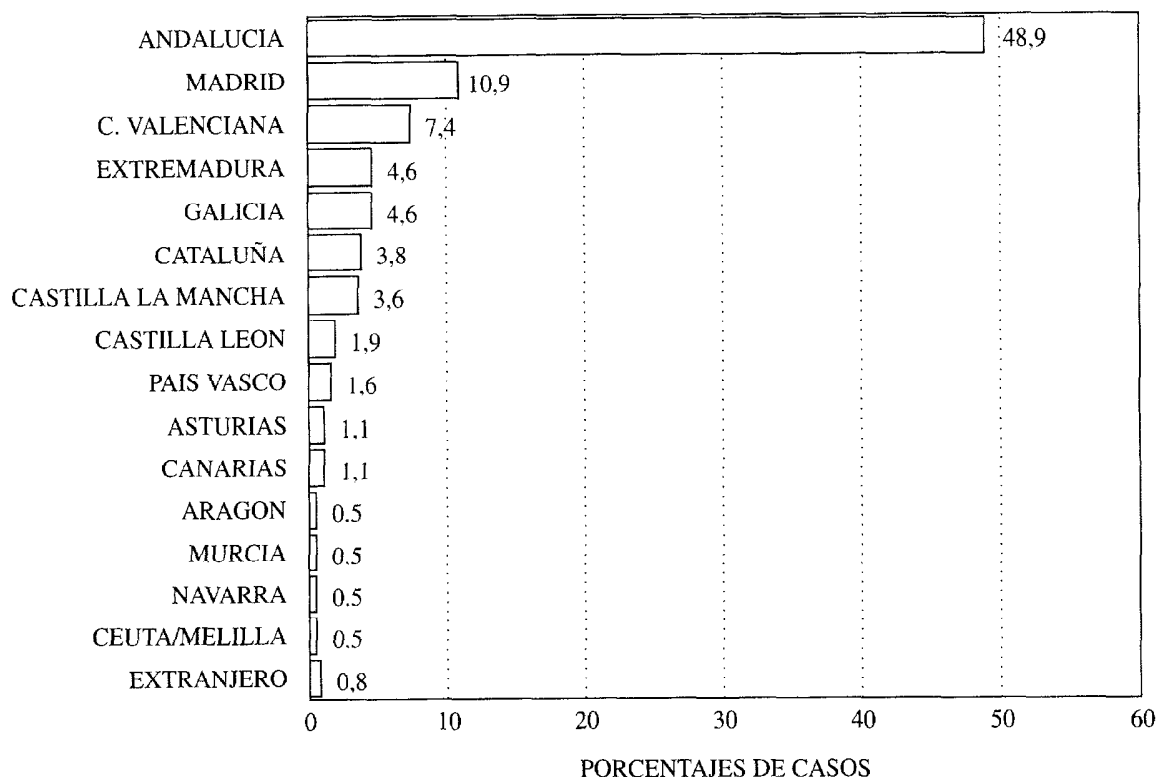

Nota: Porcentajes. 


\section{Aspectos clínicos}

Existen antecedentes familiares en los enfermos en un $31.1 \%$ de los casos ( $I C=26.4-$ 35.9) (tabla 2). Lo más habitual es que exista un sólo caso dentro de la familia $(78.83 \%)$, siendo los padres los más frecuentes.

Los tipos de lepra que se han presentado más frecuentemente han sido: lepromatosa (66.21\%, IC=61.3-71), dimorfa (12.6\%, IC $=9.2-16)$, tuberculoide $(10.1 \%, \quad I C=7$ -13.2) e indeterminada $(7.9 \%, \mathrm{IC}=5.1$ $-10.7)$. Se observan diferencias significativas entre sexos en la lepra lepromatosa y la lepra dimorfa, con una mayor afectación de los hombres $(\mathrm{p}<0.05)$ (figura 5$)$.

Aunque la edad media no presenta diferencias entre los enfermos de los distintos tipos de lepra, en la distribución por edad de los enfermos predomina en la lepra lepromatosa la edad de 20 a 39 años con un $50 \%$ de afectados. En el caso de la lepra dimorfa son los enfermos entre 15 y 24 años los predominantes con un $39.1 \%$. En la tuberculoide existen dos tramos de mayor frecuencia, el de 45-49 años (16.2\%) $y$ el de $15-24$ años (24.3\%).

En relación a la distribución del tipo de lepra por Comunidades Autónomas de pro-

Tabla 2

Variables clínicas principales según tipos de lepra (\%)

\begin{tabular}{|c|c|c|c|c|c|}
\hline & Indeterminada & Tuberculoide & Lepromatosa & Dimorfa & Total \\
\hline Antecedentes familiares & 44,8 & 27 & 32,2 & 21,7 & 31,1 I.C.:26,4-35,9 \\
\hline Edad (años) & 40 & 41,7 & 39,8 & 36 & 39,6 I.C $: 37,9-41,4$ \\
\hline \multicolumn{6}{|l|}{ Sexo } \\
\hline Varones & 65,5 & 54 & 72.2 & 69,6 & 71,9 I.C $: 67,2-76.4$ \\
\hline Mujeres & 34,5 & 46 & 24,8 & 30,4 & 28.1 I.C. $23.5-32.7$ \\
\hline Emigra. previa & 8 & 12,1 & 78,8 & I & $91 . C .6-11,9$ \\
\hline \multicolumn{6}{|l|}{ Comunidad procedencia } \\
\hline Andalucía & 59.3 & 63.6 & 50,9 & 53,7 & 48.9 I.C.:43.7-54 \\
\hline Madrid & 0,6 & 3 & 12.6 & 14,6 & 10.9 I.C.: $7.7-14.1$ \\
\hline Valencia & 7,4 & 3 & 9.1 & 4,9 & 7,4 I.C.: $: 4.7-10$ \\
\hline Galicia & 0 & 9.1 & 9,7 & 0 & $4,61, C \cdot 2,4-6.8$ \\
\hline Extremadura & 3,7 & 3 & 4,8 & 23,5 & 4.7 I.C. $2,5-6.8$ \\
\hline \multicolumn{6}{|l|}{ Afectación } \\
\hline Cabeza & 5,6 & 8,4 & 69,9 & 12,9 & 63,1 I.C.:58,2-68 \\
\hline Tronco & 8,4 & 9,9 & 63.4 & 13,6 & 37,1 I.C. $32.2-42.1$ \\
\hline Extremidades superiores & 7,6 & 10,7 & 67,4 & 11 & 64.4 I.C.:59.6-69.4 \\
\hline Extremidades inferiores & 7,5 & 10,7 & 65,2 & 13,8 & 72,1 I.C.:67,5-76,7 \\
\hline Cabeza y ext. inferiores & 0 & 0 & 70.7 & 79.3 & 27 I.C.:22.5-31,6 \\
\hline Estancia (años) & 7,3 & 6 & 8,4 & 6,3 & 7.1 I.C.:6.1-8.1 \\
\hline \multicolumn{6}{|l|}{ Causa alta } \\
\hline Transferencia & 7,6 & 10.9 & 60,2 & 19 & 57.9 I.C.:52,9 62.9 \\
\hline Muerte & 7 & 7 & 77.2 & 44 & 31,1 I.C.:26,4-35,9 \\
\hline No alta & 16,7 & 16.7 & 66,7 & 0 & 3,3 I.C. $: 1,4-5,1$ \\
\hline \multirow[t]{2}{*}{ Totales } & 7.9 & 10.1 & 66,1 & 12.6 & \\
\hline & I.C.:5, $1-10,7$ & I.C.: $7-13,2$ & I.C.:61,2-71 & 1.C.:9.2-16 & \\
\hline
\end{tabular}

I.C.: Intervalo de Confianza $95 \%$ seguridad. 
Figura 5

Tipos anatomoclínicos de lepra según sexos

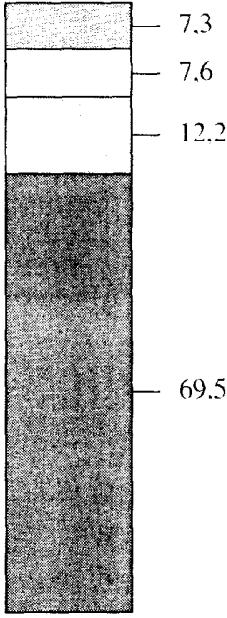

VARONES

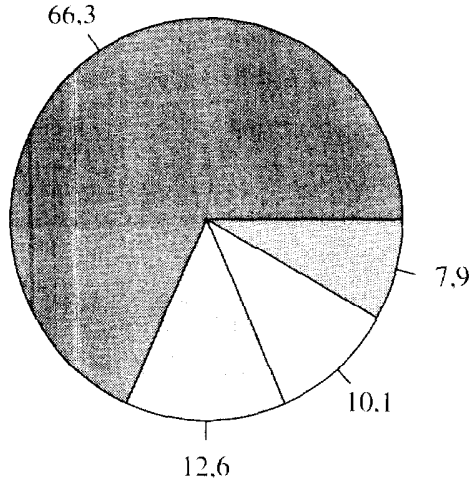

TOTALES

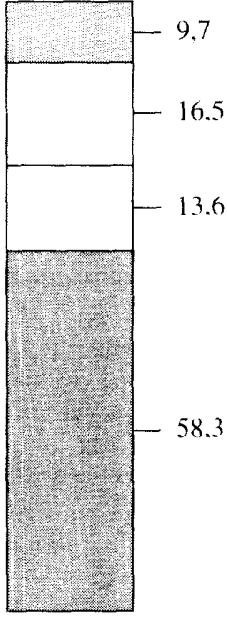

MUJERES

$\square$ LEPRA LEPROMATOSA $\square$ LEPRA DIMORFA
$\square$ LEPRA TUBERCULOIDE $\square$ LEPRA INDETERMINADA

Nota: Porcentajes.

cedencia del enfermo, se aprecia un predominio de la lepra lepromatosa en todas ellas.

En la distribución de las afectaciones en el cuerpo se ha objetivado una mayor afectación de la cabeza y ambas extremidades. En todas ellas la lepromatosa es la más frecuente. En la cabeza el número de afectados es del 63.1\% (IC=58.2-68). En el 26.9\% ha habido una sola afectación, siendo las cejas la parte más afectada.

Con afectación en el tronco se han presentado el $37.1 \%$ de los enfermos ( $\mathrm{IC}=32.2-42.1$ ).

En el $64.4 \%$ de los enfermos ha habido afectación de los miembros superiores (IC=59.6-69.4). En cuanto a la localización se da con mayor frecuencia en las manos y brazos.
Los afectados en las extremidades inferiores representan el 72.1\% (IC=67.5-76.7). En los enfermos se presentó mayoritariamente una sola afectación $(25.1 \%)$, siendo lo más habitual los glúteos y los pies.

Si estudiamos la distribución de las lesiones con aparición simultánea cn alguna partc del cuerpo, nos encontramos que lo más frecuente es la coincidencia de cabeza y extremidades inferiores con un $27.04 \%$ (IC= $=22.5-31.6$ ).

\section{La población de estudio a lo largo de la historia del centro}

La mayoría de los ingresos en el Centro tuvo lugar en los primeros 15 años de su existencia (tabla 3 ) representando el $49.2 \%$ 
Tabla 3

Población de estudio según períodos históricos

\begin{tabular}{|c|c|c|c|c|}
\hline Variables (\%) & Período inicial & Intermedio & Final & $\begin{array}{c}\text { Significación Estadística } \\
\text { Diferencias }\end{array}$ \\
\hline \multicolumn{5}{|l|}{ Sexo } \\
\hline Varón & 76,1 & 70,5 & 60 & N.S. \\
\hline Mujer & 23,9 & 29.5 & 40 & N.S. \\
\hline Edad (años) & 34,9 & 40,9 & 53,5 & $\mathrm{P}<0,05$ \\
\hline \multicolumn{5}{|l|}{ Ocupación } \\
\hline Agricultor & 43,3 & 33,8 & 14 & $P<0,005$ \\
\hline Parado & 12,2 & 19,8 & 48 & $P<0.05$ \\
\hline Minería/construc. & 11,1 & 10,3 & 6 & N.S. \\
\hline No cualificados & 9,4 & 10,3 & 4 & N.S. \\
\hline Emigración previa & 13,3 & 4,4 & 6 & N.S. \\
\hline \multicolumn{5}{|l|}{ Comunidad procedencia } \\
\hline Andalucía & 56.1 & 44,8 & 34 & $\mathrm{P}<0,005$ \\
\hline Madrid & 6,7 & 13,2 & 20 & $P<0,05$ \\
\hline Extremadura & 1.2 & 5.8 & 8 & $P<0.05$ \\
\hline Valencia & 10 & 5,1 & 4 & N.S. \\
\hline Galicia & 3,9 & 3,7 & 10 & N.S. \\
\hline \multicolumn{5}{|l|}{ Tipos clínicos } \\
\hline Lepromatosa & 73,3 & 59,6 & 58 & N.S. \\
\hline Tuberculosa & 11,6 & 9,6 & 6 & N.S. \\
\hline Indeterminada & 6,1 & 8,8 & 12 & N.S. \\
\hline Dimorfa & 6,1 & 18,3 & 20 & N.S. \\
\hline Estancia (años) & 9,9 & 5,2 & 2,2 & $\mathrm{P}<0.05$ \\
\hline \multicolumn{5}{|l|}{ Causa alta } \\
\hline Muerte & 39,4 & 25,7 & 16 & $P<0,05$ \\
\hline Transferencia & 49,4 & 60,3 & 82 & $P<0,05$ \\
\hline No alta & 2,8 & 4,4 & 2 & N.S. \\
\hline \multirow[t]{2}{*}{ Totales } & 49,2 & 37,1 & 13,6 & $\mathrm{P}<0,05$ \\
\hline & I.C.:44-54.3 & I.C.:32,2-42,5 & I.C.: $10,1-17,1$ & \\
\hline
\end{tabular}

IC $=$ Intervalo de Confianza 95\% seguridad.

N.S.= No significativo.

del total de enfermos. Este período se caracteriza por presentar un patrón más característico del enfermo leproso en el inicio de la época antibiótica: Una elevada mortalidad (39'4\%) con estancia superior a la de los períodos siguientes (9'9 años como media) y un predominio de las formas más graves (lepromatosa $73^{\prime} 3 \%$ ), en varones $\left(76^{\prime} 1 \%\right)$, jóvenes (34'9 años), agricultores (43’3\%) y andaluces $\left(56^{\prime} 1 \%\right)$.

La edad aumenta en los siguientes períodos de manera significativa así como el por- centaje de mujeres y parados. Andalucía y Valencia disminuye el número de ingresos y aumentan los ingresos de Extremadura, Madrid y Galicia.

A lo largo de los tres períodos disminuye discretamente la lepra lepromatosa, aunque siguc predominando significativamente, y aumentan las formas dimorfas.

La estancia disminuye a menos de la mitad y el número de defunciones a la tercera parte, produciéndose en la mayoría de los 
casos $(82 \%)$ transferencia a otros tratamientos no hospitalarios.

La salida del centro ocurrió globalmente por transferencia (57.9 IC:52.9-62.9) y por muerte (31.1\% IC:26.4-35.9). Un 3.3\% de los casos permanecían en el Centro por razones sociales a fecha de 1/6/1996. (figura 6).

\section{DISCUSIÓN}

En todo el estudio predominan los hombres (3/1 al principio 1'5/1 al final) a pesar del aumento progresivo de la edad al ingreso. La población estudiada ha presentado una característica distribución de edad y sexo que ha evolucionado a lo largo del tiempo; la edad de los enfermos ha aumentado en el momento del primer diagnostico, y la diferencia porcentual entre hombres y mujeres ha disminuido. Se mantienen las formas lepromatosas como predominantes, afectando más a hombres $\left(72{ }^{\prime} 2 \%\right)$ como el resto de las formas multibacilares. Esta mayor afectación y más grave del hombre se mantiene con discretas diferencias en series publicadas de España ${ }^{12}, 20-22$, procedentes de ámbitos con distintas prevalencias comunitarias. La Guía de la OMS lo refiere como un hecho revisado en países afectados. En las zonas más prevalentes y con casos mas graves la diferencia es mayor ${ }^{24}$, y aunque existen países latinoamcricanos quc han publicado series en las que no se aprecian diferencias significativas por $\operatorname{sexos}^{25-28}$, en estudios más nu$\operatorname{merosos}^{29}$ se mantienen esas diferencias. Revisiones amplias refieren algunas zonas africanas en donde la mujer llega a ser más afectada $^{30}$, pero no dejan de ser una excepción según los autores que han revisado la relación de sexo y enfermedad ${ }^{4}$.

Así pues, la mayor afectación de los varones en nuestro estudio refleja una de las características de la enfermedad de Hansen, que todavía actualmente mantiene la contro-

Figura 6

Causas de alta reflejada en la historia clínica

TRANSFERENCIA

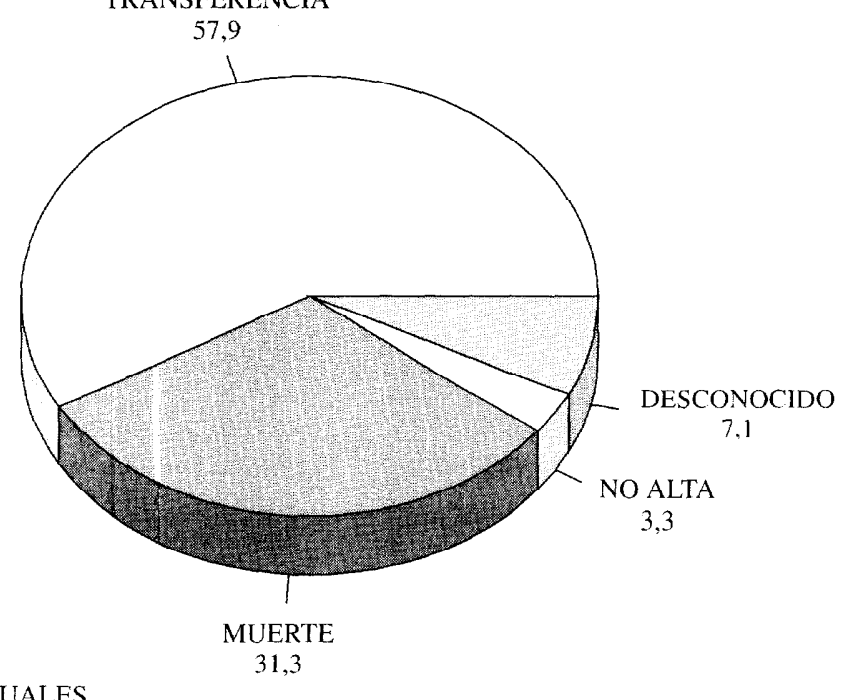

VALORES PORCENTUALES

Notu: Porcentajes. 
versia respecto a su origen inmunológico ${ }^{31}$, fisiológico-hormonal o ambiental ${ }^{4}$.

Respecto a la edad, se ha observado un aumento de la misma en los nuevos ingresados, como sucede habitualmente cuando se introducen tratamientos eficaces en el control de una enfermedad -que es lo que pasó en nuestra serie-. Esto también se ha observado en zonas de mayor prevalencia en Sudamérica ${ }^{29}$, en las que se ha hecho seguimiento de las cohortes de cnfermos y de la incidencia de la enfermedad.

Las edades más afectadas son las comprendidas entre los 20-35 años, con una edad media total de 39 años (en el período inicial de máxima incidencia en la comunidad fue de 34'9 años y al final de 53), que es similar a estudios comunitarios de nuestro país ${ }^{22}$ y discretamente superior a estudios poblacionales de países muy prevalentes ${ }^{23}$, en los que también se han observado patrones bimodales entre los 10 y 50 años ${ }^{13}$. La escasa afectación de niños es coherente con los datos publicados a este respecto por otro Hospital español ${ }^{32}$, y parece ser consecuencia de los cuidados de protección y aislamiento que se venían practicando con recién nacidos de madres leprosas. El resto de las características sociodemográficas del enfermo ingresado coinciden con el patrón social de la enfermedad descrito en estudios específicos y comunitarios en España ${ }^{33}$, y en otros países de mayor prevalencia ${ }^{4}$; enfermos con altos índices de analfabetismo, sin ocupación o con ocupaciones mal remuneradas, en agricultura o construcción, y que viven en áreas deprimidas (Andalucía) o emigran a las grandes ciudades (Madrid). Todo ello coherente con la asociación de la enfermedad a la pobreza en todo el mundo ${ }^{2.3}$ y con el terrible handicap de producir más pobreza a través de la estigmatización, la incapacidad y la afectación familiar. La existencia de más solteros en el centro no ha podido ser contrastada, pero su mayor frecuencia en un centro asistencial es lógica, teniendo en cuenta además la introducción de tratamiento en los años 1945-48 y por tanto, la posibilidad de atención domiciliaria, cuando los cuidados estaban garantizados por la familia.

Por último, en este sentido hay que reseñar que la evolución histórica del patrón social de los ingresados, incrementándose los parados y los residentes de la Comunidad de Madrid, también es coherente con los movimientos migratorios del período de estudio, ampliamente documentados en una publicación reciente ${ }^{33}$. Otro aspecto poco relevante, como el bajo porcentaje de enfermos que habían emigrado a otros países antes de su ingreso en el centro, descarta la posibilidad de importación de la enfermedad de otras áreas, hecho que está sobradamente probado en revisiones amplias del tema $a^{5.6}$ y que demuestra la importancia de nuestra endemia autóctona ${ }^{20-23}$. Las formas anatomoclínicas multibacilares (lepromatosas, dimorfas) han sido las más prevalentes en el centro en todo momento, han sido las que mayores estancias han ocasionado (7'3 años) las de mayor letalidad $\left(60^{\prime} 6 \%\right)$ y las más frecuentes en los enfermos que procedían de las comunidades más afectadas. Esto, que refleja lo que sucede en estudios comunitarios españoles ${ }^{12,21,22}$ y en extensas revisiones históricas de países más afectados ${ }^{29}$, se ha suavizado a lo largo de estudio, probablemente por la introducción de pautas antibióticas en la comunidad y por haberse mejorado su eficacia con antibióticos a los que el germen no ha creado resistencia ${ }^{8.34}$.

Otro hallazgo importante, registrado en la anamnesis de los enfermos, ha sido la existencia de antecedentes familiares $\left(37^{\prime} 7 \%\right)$, siendo los padres los más referidos como enfermos previos. Este hecho que se corrobora en estudios sociales sobre la cohorte de leprosos en España ${ }^{22}$ y que viene referido en revisiones desde el siglo $\mathrm{XIX}^{24}$ en todo el mundo, ha sido motivo para investigar un posible genotipo o fenotipo hereditario vinculado a la inmunidad general ${ }^{31}$, al $\operatorname{sexo}^{4}$ o a genes específicos ${ }^{35}$, sin que hasta ahora se hayan podido demostrar modelos genéticos mendelianos de vulnerabilidad a la enferme- 
dad, ni que existan genes específicos que determinen la penetración familiar de la enfermedad ${ }^{35}$, aún en países de alta prevalencia (Brasil, China, Vietnam). Quedan pues los factores medioambientales y de relación exclusivamente, como posibles causas de esta presentación.

Respecto a la localización de las lesiones en la exploración del enfermo, aparece la cara (cejas) y extremidades inferiores (glúteos) y superiores (brazos) como las partes más comúnmente afectadas de cabeza y extremidades. Esto, que depende de los tipos histológicos de presentación ${ }^{3}$ también se refiere en amplias definiciones epidemiológicas de caso $^{35.6 .36}$ en revisiones recientes ${ }^{37}$ y en estudios de seguimiento riguroso de áreas endémicas $^{25}$, mostrándose así un especial tropismo del bacilo de Hansen por la piel y los nervios periféricos de la cara y extremidades, con persistencia de estas localizaciones como más frecuentes, sea cual sea la comunidad de procedencia del enfermo.

El pronóstico y la evolución de los enfermos no han sido registrados homogéneamente en las historias clínicas y se ha evaluado indirectamente a través del motivo de alta y la estancia en años en el centro. La estancia ha ido disminuyendo (de 10 años ha pasado a 2 en el último período), parece que paralelamente a la introducción de la dapsona en los años 45-50, la clofazimina en los 60, la rifampicina en $\operatorname{los} 70$ y la poliquimioterapia en $1981^{8,34}$ por los comités de expertos internacionales, y tal y como ha sucedido en otras series comunitarias ${ }^{29}$. Por otra parte se han comunicado períodos de internamiento entorno a 1-3 años en la mayoría de los enfermos $(60 \%)$ en un amplio estudio comunitario realizado en la cohorte de enfermos de nuestro país ${ }^{33}$, lo que plantea una similitud de las cifras obtenidas por nosotros en el período final del centro. La letalidad, alta inicialmente, ha evolucionado paralelamente a la estancia y por tanto parece haber estado mediatizada a la existencia de formas clínicas multibacilares y de un tratamiento preciso y eficaz con- tra las mismas (poliquimioterapia). Así, aunque la letalidad de un $30 \%$ global en todo el período de estudio es similar a la encontrada en series comunitarias españolas ${ }^{20}$, su evolución de un $39 \%$ en período inicial a un $16 \%$ al final del estudio, inversa que la transferencia a centros no monográficos (del $49^{\prime} 4 \%$ al principio a un $82 \%$ al final) es un dato mas para confirmar el control de la enfermedad.

Podemos concluir pues, que tras el estudio de los ingresados en el centro éste se comporta como una «Atalaya» desde la que podemos observar el comportamiento más severo de la enfermedad en la comunidad, sobre todo en la época pre-multiterapia. El perfil del enfermo refleja en sus principales características, frecuencias similares a las aportadas por estudios comunitarios en distintos ámbitos y áreas.

También la frecuentación refleja la evolución histórica de la enfermedad en nuestro país en los últimos 50 años, que no es básicamente distinta a la de aquellos países con endemia autóctona — no importada - tras la introducción de la poliquimioterapia y después del esfuerzo en captación y seguimiento de los enfermos por el Sistema Nacional de Salud.

El perfil de varón joven y sumergido en círculos económicos precarios, en áreas tradicionalmente pobres y endémicas, con afectación más frecuente por formas clínicas multibacilares que predominan en las localizaciones de cabeza y miembros inferiores, se ha ido suavizando a lo largo del período de estudio hacia enfermos de ambos sexos, mayores, parados de grandes ciudades y con formas clínicas más leves.

\section{AGRADECIMIENTOS}

Agradecemos a todo el personal del Centro Leprológico de Trillo su ayuda y colaboración al aportarnos la documentación necesaria para la realización de nuestro trabajo, y muy especialmente al Director Médico, Dr. Juan Luis Gracia Ochaita, por su aseso- 
ramiento y por la revisión que ha hecho de nuestro trabajo.

También agradecemos al Dr. Rafael Magro Perteguer su apoyo y asesoramiento desde el inicio del proyecto, como profesor de la Escuela Universitaria de Enfermería.

\section{BIBLIOGRAFÍA}

1. Terencin de las Aguas J. La Lepra. Siete Días Méd 1996: 48-54.

2. Martín González V. La lepra en España 19431990 [Tesis Doctoral]. Alcalá de Henares: Universidad de Alcalá de Henares; 1995.

3. Bullock WE. La lepra. En: Mandell GC, Gordon Douglas R, Benmett JF. Enfermedades Infecciosas. Principios y Prácticas. Buenos Aires: Editorial Médica Panamericana; 1991. p. 2018-2025.

4. Ulrich M, Zulveta AM, Cáceres G, Sampson C, Pinardi MF, Rada FM, Aranzazu N. Leprosy in women. Venezuela. Soc Sci Med 1993; 37(4): 445-456.

5. Gómez López L, Marcos G, Abad JM. Lepra, Epidemiología y Medicina Preventiva y Social. En: Piédrola G, Del Rey J. Domínguez M, et al. Medicina Preventiva y Salud Pública. 9led. Barcelona: Editorial Masson-Salvat-Medicina; 1991.

6. Sanz Colomo B. Lepra. En: Farreras P. Rozman C. editores. Medicina Interna. 130 ed. Barcelona: Doyma: 1996. p. 2371-76.

7. Organización Mundial de la Salud. Comité de Expertos en Lepra. Ginebra: Organización Mundial de la Salud; 1988. Informe Técnico núm 768. p. 9-14.

8. Noorden SK. Elimination of leprosy as a public health problem. Bull World Health Organ 1995; 73 (1): $1-6$.

9. WHO. Progress towards the elimination of leprosy as a public health problem. Weekly Epidemiol Rec 1996; 20:149-156.

10. Centro Nacional de Epidemiología. Registro Nacional de Lepra. Informe de Situación a 27.9.96. Madrid: Centro Nacional de Epidemiología; 1996.

11. WHO. Global Strategy for Health for all by the year 2000. Ginebra: WHO; 1981. Serie SPT 2000. núm. 3.
12. Fernández Miranda R, Gil Fernández RM. Historia y Epidemiología de la lepra en Almería. Rev Leprol Fontilles 1987; 16 (1): 45-50.

13. WHO. Epidemiología de la lepra en relación con la lucha antileprosa. Ginebra: WHO; 1985. Informe Técnico núm. 716. p. 17-29.

14. Ministerio de Sanidad y Consumo.Dirección General de Planificación Sanitaria. Una guía para el control de la lepra. Madrid: Ministerio de Sanidad y Consumo; 1990. p. 71-90.

15. Herrera Casado A. Los Baños de Trillo. Encuentros culturales 1992. Madrid: Central Nuclear do Trillo; 1993.

16. Levy PS, Lemeshow S. Sampling of population; Methods and Applications. New York: John Wyley \& Sons.edit; 1991. p. 68-98.

17. Centers for Diseases Control. Manual EPIINFO (V6). Atlanta: Centers for Diseases Control; 1994.

18. Instituto Nacional Estadística. Clasificación Nacional de Ocupaciones de 1994. Madrid: Instituto Nacional Estadística; 1995.

19. Terencio de las Aguas J. VI Congreso Internacional de Leprología de Madrid. Rev Leprol Fontilles 1995; 20 (1): 599-601.

20. Delgado M, Rodriguez-Contreras R, Extremera F, Serrano S, Gálvez R. Aspectos epidemiológicos de la Lepra en la provincia de Jaén. Rev Clín Esp $1989 ; 185$ (2): 85-89.

21. Vivancos G, García A, Cabeza ML. Alvarado A, Díaz A, apote L. La Lepra en Santa Cruz de Tenerife: estudio de 25 años de su endemia 19601985. Rev Leprol Fontilles 1987; 16 (2): 191-197.

22. Patronato de Rehabilitación Social del Enfermo de Lepra. Ministerio de Servicios Sociales. Análisis de la realidad social del enfermo de Lepra. Madrid: Eurocolor; 1992. p. 65-78.

23. Ministerio de Sanidad y Consumo. Dirección General de Planificación Sanitaria. Una Guía para el control de la Lepra. Madrid: Ministerio de Sanidad y Consumo: 1990. p. 13-22.

24. Choudhury AM. Leprosy in Bangladesh 19841988. Quart Bibli of M Trop D 1993; 16 (2): 77-80.

25. Castaño S, Carrazana GB. Incidencia de Lepra en el Municipio de Nuevitas, Cuba. Estudio de 25 años (1968-1992). Rev Leprol Fontilles 1993: 19 (3): 298-303.

26. López ME, Carrazana GB, Castan̄o S. Indicadores epidemiológicos de la incidencia de Lepra en un distrito de salud. Rev Leprol Fontilles 1995; 20 (1): 625-644.

27. Gómez A, Cantillo L, López. S. Zamora D. Medina $\mathrm{ME}$, 'Torrez D. Foco leprógeno en San Francisco 
Libre, Nicaragua 1990. Rev Leprol Fontilles 1992; 18(5): 481-511.

28. Ferrá TM. Carrazana GB. Incidencia de la lepra en la ciudad de Camagüey, Cuba 1978-1993. Rev Leprol Fontilles 1995; 20 (1): 603-613.

29. Lombardi C. Ramírez R, Gil S. Rcinaldo E. Tendencia temporal de la detección de la lepra en el Estado de Sao Paulo (Brasil) 1934-1983. Rev Leprol Fontilles 1994: 20 (4): 359-386.

30. Nordeen Sk, The Epidemiology of Leprosy. In: Leprosy. Edimburg: Churchill Livingstone; 1985. p. $15-20$.

31. Castells A. Terencio de las Aguas J. Inmunología de la Lepra. Rev Leprol Fontilles 1992; 18 (4): 357-393.

32. Terencio de las Aguas J. Lepra en la infancia. Rev Leprol Fontilles 1994: 20 (6): 639-648.
33. Patronato de Rehabilitación Social del Enfermo de Lepra. Ministerio de Servicios Sociales. Análisis de la realidad social del enfermo de Lepra. Madrid: Eurocolor; 1992. p. 31-63.

34. Organización Mundial de la Salud. Comité de Expertos de Lepra. Ginebra: Organización Mundial de la Salud. Serie Informes Técnicos núm 768. p. 30-37.

35. Fertora MF, Borecki I. Krieger H, Beiguelman B, Rao DC. The Genetic Epidemiology of Leprosy in a Brazilian Population. Am J Hum Genet 1995; 56:1179-1185.

36. Ministerio de Sanidad y Consunno. Dirección General de Planificación Sanitaria. Una Guía para el control de la lepra. Madrid: Ministerio de Sanidad y Consumo; 1990. p. 33-48.

37. Terencio de las Aguas J. Pasado y Presente de la Lepra. Rev Leprol Fontilles 1991; 17 (3): 223-225. 